\title{
Autonomie oder Sezession - Das Selbstbestimmungsrecht der Sri Lanka-Tamilen in der Diskussion um eine neue Verfassung Sri Lankas ${ }^{1}$
}

\author{
Von Frank-Florian Seifert, Würzburg
}

\section{Einleitung}

"If the Sinhala nation fails to redeem itself from the grip of racism and continues its repression against the Tamils, we have no alternative other than to secede and form an independent Tamil State." Mit dieser neuerlichen Drohung einer Sezession zur Errichtung eines sri lanka-tamilisch dominierten Staates "Tamil Eelam" auf sri lankischem Staatsgebiet begleitete Velupillai Prabhakaran, Führer der "Liberation Tigers of Tamil Eelam" (LTTE), Ende November 2000 sein im Rahmen der alljährlichen "National Heroes Day Message" unterbreitetes Gesprächsangebot an die sinhalesisch dominierte Regierung Sri Lankas. ${ }^{3}$ Vermittler zwischen Regierung und LTTE-Guerilla - den Vertretern der Parteien des seit drei Jahrzehnten mit Waffengewalt ausgetragenen ethnischen Konfliktes - werden dagegen mit der Aussage zitiert, LTTE-Führer Velupillai Prabhakaran habe bereits "changed his earlier stance for a separate state to an autonomous region within a[n] united Sri Lanka". 4

Gegenstand dieses Beitrages ist die bereits 1995 von der Regierung Sri Lankas angestossene Verfassungsreform, unter anderem mittels eines "devolution package" ein Autonomie-Konzept zu verwirklichen, welches der aus den Reihen der Sri Lanka-Tamilen erhobenen Sezessionsforderung entgegengestellt und damit letztlich der Beilegung des sinhale-

Der folgende Beitrag schliesst sich an den einführenden Artikel des Autors zum völkerrechtlichen Problem der Sezession im Kontext des Konfliktes um das Selbstbestimmungsrecht der Sri LankaTamilen in Heft 1 an, vgl. Seifert, F.-F., Die Verfassungsreform in Sri Lanka unter besonderer Berücksichtigung des Selbstbestimmungsrechts der Sri Lanka-Tamilen, in: VRÜ 34 (2001), S. 4862; jener erste Teil erschien auch unter dem Titel "Das Selbstbestimmungsrecht der Sri LankaTamilen" in Südasien 20 (2000), Nr. 5, S. 17-25.

Zit. nach: LTTE Headquarters, Tamil Eelam, Press release 27.11.2000 (http://www.eelam.com/ freedom_struggle/ltte_press_releases/2000/november/27.html, AVL: 29.11.2000).

Zu den Gesprächen zwischen Regierung und LTTE vgl. The Sunday Times, The Political Column, 03.12.2000 (http://www.lacnet.org/suntimes/001203/pol.html, AVL: 04.12.2000), 10.12.2000 (http://www.lacnet.org/suntimes/001210/pol.html, AVL: 11.12.2000).

4 Zit. nach: The Sunday Times, The Political Column, 26.11.2000 (http://www.lacnet.org/suntimes/ 001126/pol.html, AVL: 27.11.2000). 
sisch - sri lanka-tamilischen Konfliktes dienen soll. Die Errichtung eines unabhängigen sri lanka-tamilischen Staates durch Sezession ist eine mögliche Verwirklichungsform des Selbstbestimmungsrechts des Volkes der Sri Lanka-Tamilen. Eine neue Verfassung Sri Lankas muss sich mit dieser Sezessionsforderung auseinandersetzen. Das Selbstbestimmungsrecht der Völker erfährt in der Darstellung der Verfassungsreform in Sri Lanka in diesem Beitrag daher eine besondere Berücksichtigung.

Im Folgenden schliessen sich einer Beschreibung des sinhalesisch - sri lanka-tamilischen Konfliktes (II) Ausführungen zum Selbstbestimmungsrecht der Völker (III) und zum konkreten Selbstbestimmungsfall der Sri Lanka-Tamilen (IV) an. Sodann werden der Verlauf der 1995 angestossenen Verfassungsreform dargestellt (V) und danach die Regelungsgegenstände des aktuellen Verfassungsvorschlages auf das Selbstbestimmungsrecht der Sri Lanka-Tamilen bezogen (VI).

\section{Der sinhalesisch - sri lanka-tamilische Konflikt}

Lebten im Zeitpunkt der letzten offiziellen Volkszählung in Sri Lanka 1981 etwa 14.846.000 Menschen, wuchs die Bevölkerung auf etwa 18.553.000 Menschen im Jahr 1996 an. ${ }^{5}$ Die Bevölkerung - "ethnisch höchst heterogen aber keineswegs ethnisch integriert" $^{, 6}$ - setzt sich aus vier wesentlichen Gruppen zusammen, wobei die Sinhalesen mit 74 v.H. die Bevölkerungsmehrheit bilden. Die Angehörigen der sinhalesischen Bevölkerungsgruppe besiedeln vornehmlich das Küstengebiet und das Bergland im Südwesten der Insel, sprechen Sinhala und sind ganz überwiegend buddhistischen Glaubens. In der Vorstellungswelt der Sinhalesen sind Religionszugehörigkeit und Existenzberechtigung auf der Insel untrennbar miteinander verknüpft. Obwohl die Bevölkerungsmehrheit, lastet den Sinhalesen ein "Minderheitenkomplex" an, der sich auf die Präsenz von etwa 60 Millionen Tamilen im benachbarten indischen Bundesstaat Tamil Nadu und die Sri Lanka-Tamilen im vermeintlich "eigenen" Land gründet.

Die Sri Lanka-Tamilen stellen 11 v.H. der Bewohner Sri Lankas und besiedeln vornehmlich das nordwestliche Küstengebiet, sprechen Tamil und sind ganz überwiegend hinduistischen Glaubens. Die Sri Lanka-Tamilen betrachten ihre Siedlungsgebiete im nordwestli-

Nach zwei Jahrzehnten ist nunmehr eine neue Volkszählung mit dem Datum des 28.06.2001 als Stichtag geplant, erwartet wird eine Bevölkerung von etwa 19,4 Millionen Menschen.

6

Rösel, J., Der Bürgerkrieg auf Sri Lanka - Der Tamilenkonflikt: Aufstieg und Niedergang eines singhalesischen Staates, Baden-Baden, 1997, S. 25. 
chen Küstengebiet Sri Lankas als "traditional Tamil homeland". 7 Das Konzept eines "traditional Tamil homeland" stützt sich auf die Existenz eines tamilischen Königreiches vom Beginn des 13. Jahrhunderts an im Norden Sri Lankas, welches Portugal als erste Kolonialmacht Anfang des 17. Jahrhunderts seiner Herrschaft unterwarf. Die britische Kolonialherrschaft änderte entscheidend die Bevölkerungsstruktur. Zu Beginn des 19. Jahrhunderts wurden Tamilen aus Südindien als Plantagenarbeiter angeworben, die "Indian-" oder "Estate-Tamils"; vorzuziehen ist allerdings die neutrale Bezeichnung dieser Bevölkerungsgruppe als "Hochland-Tamilen", da sie das zentrale Bergland der Insel besiedelt. Die Hochland-Tamilen machen etwa 7 v.H. der Gesamtbevölkerung Sri Lankas aus. Obwohl gleichfalls tamilsprachig und hinduistischen Glaubens, lassen sich die Hochland-Tamilen auch aufgrund ihrer eigenen Identität deutlich von den Sri Lanka-Tamilen abgrenzen. Deren Autonomie- und Sezessionsbestrebungen wurden von den Hochland-Tamilen nie unterstützt.

Die vierte wesentliche Bevölkerungsgruppe stellen mit 7 v.H. die Muslime Sri Lankas. Überwiegend tamilsprachig, besiedeln sie vornehmlich das östliche Küstengebiet Sri Lankas. Auch die Muslime unterstützten die Errichtung eines unabhängigen sri lanka-tamilisch dominierten Staates zu keiner Zeit, befürchten sie doch, andernfalls zu einer "Minderheit in einer Minderheit" zu werden und dadurch jegliches politisches Gewicht zu verlieren.

Auch ethnische Konflikte um das Selbstbestimmungsrecht der Völker lassen sich mittels bestimmter Merkmale - unter anderem nach dem Konfliktgegenstand, den Konfliktparteien, deren Verhältnis zueinander, der Unterstützung der Konfliktparteien durch Dritte und durch die Versuche zur Beilegung des Konfliktes - beschreiben, was unter Berücksichtigung des hier vorgegebenen Rahmens und räumlich Möglichen nunmehr erfolgt. ${ }^{8}$

Die Forderung der Errichtung eines eigenständigen sri lanka-tamilisch dominierten Staates - der nach Vorstellung seiner Verfechter "Tamil Eelam" heissen soll - unter Berufung auf das Selbstbestimmungsrecht der Völker ist die gegenwärtig letzte Phase einer Entwicklung des Konfliktgegenstandes, die mit dem Bestreben der Tamilen um eine stärkere Beteiligung innerhalb der kolonialen Gesetzgebung am Ende der britischen Kolonialherrschaft ihren Anfang nahm. Nach der Unabhängigkeit Ceylons 1948 forderten Vertreter ceylon-tamilischer Interessen alsbald gleiche sprachliche und religiöse Rechte, gefolgt von einer Bewegung für eine föderale Staatsstruktur. Diese Forderungen waren eine erste Antwort auf die

Vgl. dazu die Darstellungen bei Seifert, F.-F., Das Selbstbestimmungsrecht der Sri Lanka-Tamilen zwischen Sezession und Integration, Heidelberg, 2000, S. 328 f, 337-342 (Darstellungen 2, 3, 10-15 in Anhang 2).

8 Vgl. für eine ausführliche Darstellung der Genese des Konfliktes nur Rösel, J., op.cit. (Fn. 6); auch Keller, W. (Hrsg.), A Decade of Conflict - Sri Lanka 1983-1993, Dortmund, 1993; Neelsen, J., Das "Devolution Package" der Regierung von Sri Lanka - Modell zur Lösung des ethnischen Konfliktes?, in: Südasien 20 (2000), Nr. 2-3, S. 20-32 . 
mit der Verfassung von 1972 abgeschlossene "Sinhalisierung" des Inselstaates in politischer, sprachlicher und - im öffentlichen Sektor - personeller Hinsicht. Die Sri LankaTamilen betrachteten sich seitdem als im Gebrauch ihrer Sprache und in der Ausübung ihrer Religion diskriminiert, zudem bedrohte die Siedlungspolitik der sinhalesisch dominierten Staatsgewalt ihr "traditional Tamil homeland", Hunderttausende Sinhalesen wurden in den nordöstlichen Peripheriegebieten angesiedelt. Sodann forderten Vertreter sri lanka-tamilischer Interessen Autonomie und gleiche Rechte im Hinblick auf Bildung und Beschäftigung. Diese Forderung hat ihre Ursache vor allem in der Bildungs- und Beschäftigungspolitik der damaligen sinhalesisch dominierten Regierungen, die zur Bevorzugung von Sinhalesen auf diesen Gebieten führte. Die von den Sri Lanka-Tamilen als sie diskriminierend empfundenen Bildungs- und Beschäftigungsmassnahmen waren massgeblicher Grund für die Entstehung militanter Organisationen innerhalb der sri lanka-tamilischen Jugend.

Die ceylon-tamilische Konfliktpartei dominierte von der Unabhängigkeit bis zur Gründung der "Tamil United Front" (TUF) die "Federal Party" (FP). Die TUF benannte sich 1976 um in "Tamil United Liberation Front" (TULF), die in der "Vaddukoddai-Resolution" im selben Jahr erstmals die Errichtung eines unabhängigen sri lanka-tamilischen Staates durch Sezession unter Berufung auf das Selbstbestimmungsrecht der Völker forderte. ${ }^{9}$ Die mit nicht-militanten Mitteln geführte Sezessionsbewegung verlor zu Beginn der achtziger Jahre jedoch die Führungsrolle, welche von einer Vielzahl bereits in den siebziger Jahren gegründeten militanten Gruppen übernommen wurde. Die Guerillaorganisation LTTE, 1972 als "Tamil New Tigers" gegründet, schaltete alle rivalisierenden Gruppen aus und dominiert seit 1986 die (auch) mit militanten Mitteln geführte Sezessionsbewegung. Weitere Verfechter sri lanka-tamilischer Interessen stellen den Alleinvertretungsanspruch der LTTE zwar in Frage. Unbestritten aber ist die LTTE eine der mächtigsten Guerillaorganisationen der Welt, ohne deren Zustimmung eine jede neue Verfassung mit dem Ziel, den Konflikt beizulegen, wirkungslos bleiben wird.

Das Verhältnis der Konfliktparteien zueinander wurde mit Beginn des mit militanten Mitteln geführten Guerillakampfes sri lanka-tamilischer Jugendorganisationen ab Mitte der siebziger Jahre zunehmend gewalttätiger. Seitdem kam es immer wieder zu Ausschreitungen zwischen Sinhalesen und Sri Lanka-Tamilen, deren trauriger Höhepunkt der sogenannte "Schwarze Juli" des Jahres 1983 war, in dem bei Ausschreitungen Hunderte - wenn nicht Tausende, die Zahlen gehen weit auseinander - Sri Lanka-Tamilen getötet wurden. Die Ausschreitungen gegen die sri lanka-tamilische Bevölkerung waren organisiert, einen massgeblichen Beitrag der damaligen sinhalesisch dominierten Staatsgewalt hat die gegenwärtige sri lankische Regierung mittlerweile eingeräumt. Die militärischen Auseinanderset- 
zungen zwischen den sri lanka-tamilischen Guerillaorganisationen, seit Ende der achtziger Jahre vornehmlich der LTTE, und den Sicherheitskräften der sinhalesisch dominierten Staatsgewalt forderten seit 1983 mehr als 60.000 Menschenleben. Auch wenn die LTTE ihre "Hochburg" Jaffna im Dezember 1995 aufgeben musste, wird eine militärische Lösung des Konfliktes nicht für möglich gehalten. Ob die seit Ende November 2000 beabsichtigten Friedensgespräche zwischen LTTE und sri lankischer Regierung überhaupt stattfinden und gegebenenfalls einen Erfolg haben werden, ist ungewiss.

In derartige Verhandlungen wird Indien als Regionalmacht Südasiens unmittelbar wohl nicht eingebunden sein. In Erfüllung des indisch-sri lankischen Abkommens aus dem Jahr 1987 kam es zur Präsenz einer zeitweise bis zu 100.000 Soldaten starken "Indian Peace Keeping Force" (IPKF) auf dem Staatsgebiet Sri Lankas von 1987 bis 1990, die für die Beilegung des eskalierten ethnischen Konfliktes - insbesondere für die Einhaltung eines Waffenstillstandes - sorgen sollte. Die IPKF wurde von der LTTE solange bekämpft, bis der Abzug der indischen Truppen 1990 unausweichlich wurde. Zwar erfuhr die LTTE vor allem aus dem südindischen Bundesstaat Tamil Nadu Unterstützung. Die indische Zentralregierung lehnt jedoch seit jeher einen eigenständigen sri lanka-tamilisch dominierten Staat $\mathrm{ab}$ - könnte doch ein solcher Vorbildwirkung für die zahlreichen Sezessionsbestrebungen innerhalb Indiens selbst entfalten.

Initiativen zur Beilegung des ethnischen Konfliktes hat es in der Vergangenheit zahlreiche gegeben, doch selbst wenn sie zumindest rechtliche Relevanz erlangten, blieben sie erfolglos und leisteten letztlich keinen noch so geringen Beitrag, den sinhalesisch - sri lankatamilischen Konflikt zu beenden. Mit der aktuellen Verfassungsreform besteht die Chance, den eigentlichen Konfliktgegenstand - das Selbstbestimmungsrecht des Volkes der Sri Lanka-Tamilen - derart angemessen zu berücksichtigen, dass sich dieses in den sri lankischen Staatsverband eingebunden fühlt und damit die Forderung, aus dem Staatsverband auszutreten, an Bedeutung verliert.

\section{Das Selbstbestimmungsrecht der Völker}

Das Selbstbestimmungsrecht der Völker gilt in der völkerrechtlichen Theorie seit jeher als eines der umstrittensten völkerrechtlichen Prinzipien - schon die Frage, ob das Selbstbestimmungsrecht der Völker tatsächlich nur ein politisches Prinzip ist, ein Prinzip des Völkerrechts oder ein Rechtssatz dessen - mit welcher Wirkung auch immer - oder gar ein durchsetzbarer Rechtsanspruch, ist bislang nicht geklärt, da schon keine Einigkeit darüber besteht, welcher Inhalt dem Selbstbestimmungsrecht zukommt und wer dessen Rechtsträger sein soll. ${ }^{10}$ In der völkerrechtlichen Theorie besteht wohl bislang Einvernehmen nur dar- 
über, dass der Tatbestand der Entkolonisierung eine Verwirklichungsform des Selbstbestimmungsrechts der Völker ist, der zudem zu Gewohnheitsrecht erstarkte, zwischenzeitlich jedoch an Bedeutung verloren hat. Soweit das Selbstbestimmungsrecht der Völker bereits als solches ohne weitere Differenzierung als Teil des Völkergewohnheitsrechts angesehen wird, erfolgt zur Begründung dieser Ansicht üblicherweise ein Verweis auf die Bezugnahme in der UN-Charta, den beiden UN-Menschenrechtspakten aus dem Jahr 1966 und der sogenannten "Friendly Relations Declaration” von 1970.

Die völkerrechtliche Praxis - sowohl die der Staaten als auch die der Vereinten Nationen hat tatsächlich ein Selbstbestimmungsrecht der Völker ausserhalb des Entkolonisierungsprozesses trotz derartiger Bezugnahmen nie anerkannt. ${ }^{11}$ Jedenfalls dann, wenn das Selbstbestimmungsrecht eines Volkes auf Sezession verengt wird, ist dies nur zu verständlich. Eine Sezession entzieht einer Staatsgewalt unmittelbar deren Machtbasis, Staatsgebiet und Staatsvolk. Dieser Befund wurde erst durch den Kosovo-Konflikt bestätigt, in dem die völkerrechtliche Praxis mit allen Mitteln die territoriale Integrität Serbiens aufrechterhalten hat, die Kosovo-Albaner dabei allein auf ein "inneres Selbstbestimmungsrecht" verwies, welches mit einem Verbleib im serbischen Staatsverband einhergeht.

Wird ein Selbstbestimmungsrecht der Völker anerkannt, ist zunächst der Inhalt des Selbstbestimmungsrechts von seinen Verwirklichungsformen abzugrenzen. Inhaltlich bedeutet das Selbstbestimmungsrecht das Recht von Völkern, frei über ihr politisches Schicksal zu entscheiden. Mittlerweile lassen sich zahlreiche Ansätze ausmachen, die dann denkbaren Verwirklichungsformen neu zu systematisieren. Üblicherweise wird jedoch noch zwischen einem externen (1) und einem internen (2) Aspekt des Selbstbestimmungsrechts unterschieden.

\section{Das äussere Selbstbestimmungsrecht der Völker}

Das äussere Selbstbestimmungsrecht "entitles a people to decide its international identity" "12 und legt damit die Verhältnisse eines Volkes zu anderen Völkern und/oder deren Staaten fest; in diesem Fall verlangt ein Volk also einen bestimmten internationalen Status oder eine bestimmte Zugehörigkeit.

Das äussere Selbstbestimmungsrecht kann offensiv mit positiver Zielsetzung geltend gemacht werden. Fallgruppen, die sich hier einordnen lassen, sind unter anderem die Situa-

Vgl. Seifert, F.-F., op.cit. (Fn. 7), S. 79-122 m.w.N.; ders., op.cit. (Fn. 1), S. 50-52.

12

Kolodner, E., The future of the right to self-determination, in: Connecticut Journal of International Law 10 (1994), S. 153-167 (S. 159). Vgl. insbesondere zum folgenden schon Seifert, F.-F., op.cit. (Fn. 1), S. 51. 
tion der Entkolonisierung, welche heute an Aktualität verloren hat. Hier sind nun aber auch die Situationen "echter" Sezessionsfälle zu verorten. Bei "echter" Sezession verliert ein Staat einen Teil seines Staatsgebietes, auf dem mit der Abtrennung ein neuer Staat entsteht oder um den ein dritter Staat sein eigenes Staatsgebiet erweitern kann. Der vom Gebietsverlust betroffene Staat bleibt mit verkleinertem Gebiet weiter bestehen, das abgetrennte Teilgebiet ist allerdings vom betroffenen Staat nunmehr unabhängig. Aus Sicht des sezedierenden Volkes lässt sich damit nach einer "Staatenunabhängigkeitsbewegung", die auf die Errichtung eines neuen Staates abzielt, und nach einer "Gemeinschaftsanschlussbewegung", die auf den Anschluss an einen dritten Staat gerichtet ist, unterscheiden. Aus Sicht der vom Gebietsverlust betroffenen Staatsgewalt lässt sich von einer "Entlassung" sprechen, wenn die Sezession mit ihrem Willen erfolgt, andernfalls - gegen den Willen der bisherigen Staatsgewalt - von einer "Losreissung". Im Gegensatz zum inneren steht diese Verwirklichungsform des äusseren Selbstbestimmungsrechts als "dynamisch" den Stabilitätskonzepten der territorialen Integrität und Souveränität der Staaten als "statisch" in einem "Spannungsverhältnis" entgegen."

Ob ein Sezessionsrecht existiert, ist in der völkerrechtlichen Theorie höchst umstritten. ${ }^{13}$ Wird ein solches bejaht, stellt sich zunächst die Frage, wer Rechtsträger eines Sezessionsrechts sein soll. Des weiteren ist problematisch, unter welchen Voraussetzungen ein Sezessionsrecht ausgeübt werden können soll. Eine der in der völkerrechtlichen Theorie vertretenen Meinungen billigt auch einem Volk im ethnischen Sinn ein Sezessionsrecht zu. Ein Volk im ethnischen Sinn könne durch eine Kombination objektiver - beispielsweise Sprache, Religion und Siedlungsgebiet - und subjektiver Kriterien - Gemeinschaftsglaube und Selbstbestimmungswille - bestimmt werden. Weit auseinander gehen allerdings die Ansichten im Hinblick auf die Bedingungen, bei deren Eintritt ein Sezessionsrecht bestehen soll. Nur dann, wenn das Volk im ethnischen Sinn in seiner Existenz bedroht wird, soll eine Sezession ausnahmsweise erlaubt sein.

Insoweit ist die Verbindung zum inneren Selbstbestimmungsrecht der Völker angedeutet. Solange der Staatsverband das innere Selbstbestimmungsrecht des Volkes im ethnischen Sinn wahrt, ist ein Sezessionsrecht ausgeschlossen. Andererseits kann verweigerte innere Selbstbestimmung in ein Recht zur Sezession umschlagen.

\section{Das innere Selbstbestimmungsrecht der Völker}

Inneres Selbstbestimmungsrecht meint zumindest "the right of an ethnic, racial or religious segment of the population in a sovereign country not to be oppressed by a discrimi- 
natory government" 14 und berechtigt darüber hinaus "a people to participate effectively in the decision-making process which affects the political, economic, social, and cultural conditions under which it lives". 15 Die Notwendigkeit, einen bestimmten Bevölkerungsteil als Rechtsträger zu qualifizieren, besteht aber grundsätzlich nicht, da das innere Selbstbestimmungsrecht dem gesamten Staatsvolk zusteht. Beim inneren Selbstbestimmungsrecht bleibt die territoriale Integrität und Souveränität des Staates als Völkerrechtssubjekt unangetastet.

Auch die Verwirklichung des inneren Selbstbestimmungsrechts ist völkerrechtlich noch nicht geklärt. Aus der völkerrechtlichen Praxis lassen sich bislang keine verbindlichen Vorgaben etwa dergestalt, staatliche Verfassungen müssten Menschenrechte und grundlegende Freiheiten sichern, Autonomie- und Demokratie-Konzepte vorsehen, herleiten. Ein anderer Befund würde auch die Rechtswirklichkeit ignorieren.

In der völkerrechtlichen Theorie ist nach verschiedentlich vertretener Ansicht ein inneres Selbstbestimmungsrecht in der Entwicklung begriffen. ${ }^{16}$ Insoweit lassen sich auch die Verwirklichungsformen innerer Selbstbestimmung weiter unterscheiden. Auf einer ersten Ebene wird sich innere Selbstbestimmung als die Sicherung von Menschenrechten und grundlegenden Freiheiten aller Staatsbürger verstehen lassen. Auf einer zweiten Ebene erfährt innere Selbstbestimmung des Staatsvolkes dann möglicherweise zwei Ausprägungen: einmal ist eine Staatsstruktur denkbar, die ein bestimmtes Autonomie-Modell beinhaltet, ferner ist denkbar, dass der Staatsstruktur demokratische Elemente innewohnen. Auch wenn in der völkerrechtlichen Theorie bereits ein "emerging right of democratic governance" ausgemacht wird, ${ }^{17}$ bleibt bislang völlig unklar, was mit "Demokratie" gemeint sein kann, allgemein anerkannte oder gar allgemein geltende Vorgaben oder Regelungen existieren auf der Ebene des Völkerrechts nicht.

Im Völkerrecht wird "Autonomie" als Befugnis von Teilgebieten eines Staates definiert, bestimmte Angelegenheiten im Wege der Selbstverwaltung, insbesondere durch den Erlass von Rechtsnormen, selbst zu regeln, ohne dass dieses Gebiet dadurch Staatsqualität erlangt. ${ }^{18}$ Durch Autonomie wird einem Teil des Staatsgebietes oder des Staatsvolkes innere ing rights, Philadelphia, 1990, S. 113.

Vgl. Franck, T., The emerging right to democratic governance, in: American Journal of International Law, 86 (1992), S. 46-91 (S. 46, 90). 
Selbstverwaltung und damit eine teilweise Unabhängigkeit vom Einfluss der bisherigen Staatsgewalt zugestanden. Die Regelung des Einzelfalles wird zum Mass der gewährten Unabhängigkeit, es gibt kein feststehendes allgemeingültiges Modell der Autonomie. Der Begriff der Autonomie wird als unbestimmter Rechtsbegriff qualifiziert, der jeweils einer konkreten Ausfüllung bedarf.

Auch wenn sich der jeweilige Umfang der Autonomie nicht abstrakt festhalten lässt, sind doch zumindest vier Arten der Autonomie unterscheidbar: funktionelle, kulturelle, Territorial- und Personalautonomie. Daneben stellt sich noch der Sonderfall des Föderalismus, der gleichfalls unter den Autonomiebegriff gefasst werden kann. ${ }^{19}$ Bei territorialer Autonomie erhält ein Gebiet dem Territorialitätsprinzip entsprechend einen Sonderstatus, der darin besteht, dass bestimmte Angelegenheiten der Regelung durch Selbstverwaltungsorgane unterliegen. Der Träger der Autonomie ist die Gebietskörperschaft. Die übertragenen Kompetenzen gehen über eine blosse kommunale und regionale Selbstverwaltung hinaus. Da sie ausserdem spezifische Probleme der von der Autonomieregelung begünstigten Gruppe betreffen, wird den sie konstituierenden Besonderheiten am ehesten entsprochen. Allen Regelungen territorialer Autonomie ist die Schaffung einer eigenen Exekutivgewalt und einer gewählten Volksvertretung gemeinsam. Territoriale Autonomie kann nur gewährt werden, wenn die von der Autonomieregelung begünstigte Gruppe eine Siedlungsgemeinschaft bildet, also mehrheitlich ein geographisch abgeschlossenes Gebiet bewohnt.

Autonomie zielt ab auf die Einschmelzung der begünstigten Gruppe(n) in einen Einheitsstaat, Föderalismus auf die Koordination und Verknüpfung der Gruppen. Die Gliedstaaten eines Bundesstaates als staatsrechtlicher Konstruktion des Föderalismus verfügen über ein gesondertes Mass an Autonomie in Form eigener lokaler Legislative, Exekutive und Judikative. Im Gegensatz zur Autonomie kennzeichnet die föderale Staatsstruktur eine bestimmte Kompetenzverteilung zwischen Bundes- und Gliedstaaten, eine vertikale Gewaltenteilung. Autonomie ist nur eine Teilordnung, Bundesstaat und Länder immer Glieder einer Gesamtordnung.

Mit dem inneren Aspekt des Selbstbestimmungsrechts wird mittlerweile ein föderales Selbstbestimmungsrecht der Völker zusammengebracht. Die Konstruktion dessen stellt wie die Autonomie auf Einzelfalllösungen ab. Als Sonderfälle des Föderalismus sind ethnischer und polyethnischer Föderalismus anzusehen. Beim ethnischen Föderalismus fällt die politi-

(Hrsg.), Selbstbestimmungsrecht der Völker - Herausforderung der Staatenwelt - Zerfällt die internationale Gemeinschaft in Hunderte von Staaten?, Bonn, 1997; ders., Autonomie und Völkerrecht - Verwirklichung des Selbstbestimmungsrechts der Völker innerhalb bestehender Staaten, Bonn, 1995. (2000), S. 285-297 (S. 295-297), der eine bundesstaatliche Struktur, nicht aber Autonomie innerer Selbstbestimmung zuordnet, was aber vom Verständnis des Begriffes "Autonomie" abhängt. 
sche mit der ethnischen Gliederung des Staatsgebietes zusammen. In der polyethnischen Föderation dagegen ist weder der Bundesstaat noch der einzelne Gliedstaat ethnisch homogen, das zahlenmässig dominierende Volk ist über mehrere Gliedstaaten verteilt und deren Grenzen folgen nicht ethnischen Kriterien.

Wird die Errichtung autonomer Strukturen auch als taugliches Mittel angesehen, ethnischen Konflikten vorzubeugen oder solche sogar beizulegen, besteht daneben doch die begründete Gefahr, mit gewährter Autonomie einer Sezession Vorschub zu leisten. Dies gilt gerade auch für eine föderale Staatsstruktur. Wegen eines derart einseitigen Verständnisses, - jede gewährte Autonomie führe notwendig zur Sezession - scheiterten bislang im Fall Sri Lankas alle Versuche, autonome Strukturen zu errichten.

\section{Das Selbstbestimmungsrecht der Sri Lanka-Tamilen}

Der sinhalesisch - sri lanka-tamilische Konflikt hat mittlerweile eine Sezessionsforderung zum Konfliktgegenstand, die folgenden Ausführungen setzen sich in der gebotenen Kürze mit einer möglichen völkerrechtlichen Begründung dieser Forderung auseinander (1). Sodann wird darauf eingegangen, inwieweit bislang ein inneres Selbstbestimmungsrecht der Sri Lanka-Tamilen zur Verwirklichung kam (2).

\section{Das äussere Selbstbestimmungsrecht des Volkes der Sri Lanka-Tamilen}

Ein unabhängiges "Tamil Eelam" wird sich nur durch Losreissung verwirklichen, die sri lanka-tamilische Staatenunabhängigkeitsbewegung wird von der sinhalesisch dominierten Staatsgewalt bekämpft.

Ob die Errichtung eines sri lanka-tamilisch dominierten Staates "Tamil Eelam" tatsächlich unter Berufung auf das Selbstbestimmungsrecht der Völker gefordert werden kann, lässt sich mit völkerrechtstheoretischen Argumenten überhaupt nur dann bejahen, wenn mit einer der in der völkerrechtlichen Theorie vertretenen Ansichten die Existenz eines Sezessionsrechts im Ausnahmefall anerkannt wird. Wird dieser Ansicht gefolgt, lässt sich zwar die Rechtsträgereigenschaft der Sri Lanka-Tamilen als Volk im ethnischen Sinn problemlos bejahen. ${ }^{20}$ Über die erwähnte Kombination objektiver und subjektiver Kriterien qualifizieren sich die Sri Lanka-Tamilen als selbstbestimmungs- und damit auch sezessionsberechtigtes Volk. Fraglich bleibt jedoch, ob die hohen Anforderungen, die in der völkerrechtlichen Theorie an die Voraussetzungen der Ausübung eines Sezessionsrechts gestellt werden, im Selbstbestimmungsfall der Sri Lanka-Tamilen erfüllt sind. Gerade wegen ihrer Volkszu- 
gehörigkeit müssen die Angehörigen des Volkes der Sri Lanka-Tamilen seit Erlangung der Unabhängigkeit vor mehr als fünf Jahrzehnten auf zahlreichen Gebieten eine Diskriminierung erdulden. Besonders schwerwiegend sind dabei die anlässlich der militärischen Auseinandersetzungen der sinhalesisch dominierten Regierung zur Last fallenden Verletzungen der Menschenrechte und grundlegenden Freiheiten. Aber auch eine eingehendere Analyse der Diskriminierung des sri lanka-tamilischen Volkes ergibt, dass das in der völkerrechtlichen Theorie aufgestellte Mass nicht mehr hinnehmbarer Diskriminierung - die Aufhebung der Existenzgrundlagen des Volkes - noch nicht erreicht ist. ${ }^{21}$ In der völkerrechtlichen Theorie würde - selbst unter Zugrundelegung der sezessionsfreundlichsten Ansichten - ein Sezessionsrecht des Volkes der Sri Lanka-Tamilen abgelehnt. Nicht verschwiegen werden soll an dieser Stelle, dass die völkerrechtliche Theorie den Selbstbestimmungsfall der Sri Lanka-Tamilen weitestgehend genau so ignoriert wie die völkerrechtliche Praxis.

\section{Das innere Selbstbestimmungsrecht des Volkes der Sri Lanka-Tamilen}

Im Hinblick auf die oben genannte zweite Ebene innerer Selbstbestimmung mit ihren Ausprägungen demokratisch legitimierter Staatsgewalt und gewährter autonomer Strukturen lässt sich festhalten, dass dem Volk der Sri Lanka-Tamilen bislang innere Selbstbestimmung vorenthalten blieb. ${ }^{22}$ Beispiele für nur mangelhafte demokratische Strukturen sind nicht nur das Referendum des Jahres 1982 als Ersatz der Parlamentswahlen oder die Wahlen zum Provinzrat der Nordöstlichen Provinz im Jahr 1988. Zu bedenken ist, dass auf dem nordöstlichen Staatsgebiet Sri Lankas seit mehr als zwei Jahrzehnten militärische Auseinandersetzungen stattfinden, die kaum ein Klima entstehen lassen, von etwaigen demokratischen Rechten überhaupt nur Gebrauch zu machen. Gerade die sri lanka-tamilische Bevölkerung ist an der Ausübung dieser allein schon faktisch gehindert und von einer demokratischen Willensbildung weitestgehend ausgeschlossen. Bislang sind zudem jegliche Versuche gescheitert, funktionierende autonome Strukturen, die zumindest auch das sri lankatamilische Volk begünstigen sollten, zu errichten. Rechtliche Relevanz erlangten kurzzeitig allein das beabsichtigte System der "District Development Councils" zu Beginn der achtziger Jahre und das der "Provincial Councils", welches auf der 13. Verfassungsänderung aus dem Jahr 1987 basiert, welche wiederum dem indisch-sri lankischen Abkommen desselben Jahres folgte. Aus mehreren Gründen waren jedoch auch diese Autonomie-Konzepte im nordöstlichen Staatsgebiet nie funktionsfähig.

Jedoch kann aus der Verweigerung innerer Selbstbestimmung auf deren zweiter Ebene Demokratie und Autonomie - ein Umschlagen in ein Recht zur Sezession ohnehin nicht

21

22

Vgl. Seifert, F.-F., op.cit. (Fn. 7), S. 177-238 m.w.N.; ders., op.cit. (Fn. 1), S. 57-59.

Vgl. Seifert, F.-F., op.cit. (Fn. 7), S. 225-233 m.w.N.; ders., op.cit. (Fn. 1), S. 61 f. 
hergeleitet werden. ${ }^{23}$ Selbst die in der völkerrechtlichen Theorie vertretenen sezessionsfreundlichsten Ansichten verneinen in diesem Zusammenhang die rechtlich begründbare Möglichkeit einer Sezession - dies selbst im europäischen Kontext - was das Agieren der völkerrechtlichen Praxis im Kosovo-Konflikt auch bestätigte. Allein die Verweigerung innerer Selbstbestimmung auf deren erster Ebene - Menschenrechte und grundlegende Freiheiten - kann ausnahmsweise zu einem Sezessionsrecht führen.

\section{Die Verfassungsreform seit 1995}

Das Wahljahr 1994 veränderte die innenpolitische Lage Sri Lankas massgeblich: Gerade aus den Parlaments- und Präsidentschaftswahlen ging das Bündnis der "People's Alliance" (PA) unter Führung der "Sri Lanka Freedom Party" (SLFP) als Sieger hervor und verwies damit nach fast zwei Jahrzehnten die bis dahin regierende "United National Party" (UNP) in die Opposition. Die Wahlkämpfe wurden seitens der PA unter anderem - aber nicht vordergründig - mit dem Bestreben nach einer Lösung des ethnischen Konfliktes zwischen Sinhalesen und Sri Lanka-Tamilen geführt. Die von der Regierungskoalition seit 1995 unterbreiteten Vorschläge für eine Verfassungsreform - die Annahme einer neuen Verfassung, nunmehr der vierten seit Erlangung der Unabhängigkeit - sind Bestandteil einer Strategie zur Konfliktbeilegung, die zugleich auf die militärische Vernichtung der LTTEGuerilla abzielt. Einschliesslich des Gesetzes zur Verfassungsreform vom 03.08.2000 unterbreitete die sri lankische Regierung seit 1995 fünf im weiteren Verlauf jeweils vervollständigte Vorschläge für eine Verfassungsreform.

\section{Die "Proposals for devolution of power in Sri Lanka" vom 03.08.1995}

Die von der sri lankischen Regierung am 03.08.1995 vorgelegten "Proposals for devolution of power in Sri Lanka" waren noch kein vollständiger neuer Verfassungstext, zunächst wurde allein das "devolution package", das Autonomie-Konzept, als "Kern" der zukünftigen Verfassung veröffentlicht. ${ }^{24}$

Das Autonomie-Konzept sah die Errichtung einer "Union of Regions" vor, die die Republik Sri Lanka - "united and sovereign" - bilden sollte. ${ }^{25}$ Dementsprechend würde - als

24 Text unter: LAcNet (http://www.lacnet.org/srilanka/politics/devolution/proposal.html, AVL: 24.08.2000). Vgl. auch Seifert, F.-F., op.cit. (Fn. 7), S. 260-263 m.w.N.

25

Vgl. Art. 9.1.a., 9.1.b. Regierungsvorschlag 03.08.1995. Beabsichtigt war die Errichtung eines "effective constitutional framework for the sharing of power with the regions based on an inter- 
"framework" der "devolution" - die "legislative power of the People" durch das (Zentral-) Parlament, die "Regional Councils" und das Staatsvolk selbst mittels Referenden ausgeübt, die "executive power of the People" durch den Präsidenten der Republik - "acting on the advice of the Prime Minister and the Cabinet of Ministers" - und die Gouverneure "acting on the advice of the respective Chief Ministers and Regional Boards of the Ministers" - der Regionen. ${ }^{26}$

Die "substance of devolution" zugunsten der Regionen sollte sich durch eine "exclusive legislative and executive competence within the devolved sphere" auszeichnen. ${ }^{27}$ Letztere wurde durch 46 in einer "Regional List" aufgeführte Bereiche umschrieben. ${ }^{28}$ Die dem Zentrum verbleibenden Kompetenzen auf 58 Gebieten waren in einer "Reserved List (Central Government List)" festgehalten. ${ }^{29}$ Als "unit" innerhalb der "structure of devolution" sollten "regions" eingerichtet werden. Bereits im ersten Regierungsvorschlag wurde in Aussicht gestellt, dass eine der Regionen "would be constituted by redemarcating the existing boundaries of the present North-East Province in full consultation with a view to reconciling Sinhala, Tamil and Muslim interests". Institutionen einer "unit of devolution" sollten der Regional Council als auf fünf Jahre gewähltes Legislativorgan mit einem "Speaker" und "Deputy Speaker", der "Chief Minister" und der "Board of Ministers" als dem "Regional Council" kollektiv verantwortliches Exekutivorgan und der "Governor" sein. $^{31}$

nationally consistent and coherent value system", Punkt e) Präambel Regierungsvorschlag 03.08.1995.

Vgl. Art. 9.1.c., 1.2.1., 9.1.d., 1.4.2. Regierungsvorschlag 03.08.1995.

27 Vgl. Art. X Regierungsvorschlag 03.08.1995. Eine "Permanent Commission on Devolution" sollte Streitigkeiten zwischen Zentrum und Regionen oder zwischen Regionen beilegen, die Kommission "will have powers of mediation as well as adjudication", Art. 8.1. Regierungsvorschlag 03.08.1995.

Die Bereiche sind aufgeführt unter Punkt A der "Lists" im Anhang des Regierungsvorschlages 03.08.1995 (Beispiele: "2. Education and Educational Services", "16. Local Government", "24. State land and its alienation or disposal", "25. Regional police and law and order", "27. Regional public service").

Die Bereiche sind aufgeführt unter Punkt B der "Lists" im Anhang des Regierungsvorschlages 03.08.1995 (Beispiele: "1. Defence, national security, national police, and the security forces", "3. Foreign Affairs", "6. Currency and Foreign Exchange, international economic relations and monetary policy", "32. National Universities", "51. Buddhism").

Vgl. Art. 1.1. Regierungsvorschlag 03.08.1995.

31

Vgl. Art. 1.2., 1.2.1., 1.4.2., 1.3. Regierungsvorschlag 03.08.1995. 


\section{Die "Draft provisions of the constitution containing the proposals of the government of Sri Lanka relating to devolution of power" vom 16.01.1996}

Auch die von der sri lankischen Regierung am 16.01.1996 aufgestellten "Draft provisions of the constitution containing the proposals of the government of Sri Lanka relating to devolution of power" stellten noch keinen vollständigen neuen Verfassungstext dar, ${ }^{32}$ entwickelten jedoch das bereits vorgelegte devolution package in institutioneller Hinsicht weiter und ergänzten dieses um eine verfassungsrechtlich verankerte Absicherung gegen Sezession. Die eigentliche Bedeutung des Regierungsvorschlages vom 16.01.1996 lag jedoch in der beabsichtigten Abschaffung der "executive presidency" im Rahmen eines neuen Demokratie-Konzeptes zur Errichtung einer parlamentarischen Demokratie, welches neben das Autonomie-Konzept treten sollte.

\section{Der Verfassungsvorschlag vom 27.03.1997}

Im Unterschied zu den ersten beiden sich vornehmlich auf die Grundstruktur eines Autonomie-Konzeptes beschränkenden Reformvorschlägen der sri lankischen Regierung wurde erstmals der Vorschlag für eine neue Verfassung vom 27.03.1997 zu einem nahezu vollständigen Verfassungstext ausgeweitet. ${ }^{33}$ Dabei blieb das Autonomie-Konzept allerdings ausgeklammert, bestätigt wurden aber sowohl die - zugleich erweiterten - Regelungen zur Absicherung gegen Sezession als auch das Demokratie-Konzept. Von erheblicher Bedeutung war deshalb der erstmals in den Verfassungstext eingearbeitete Katalog von Menschenrechten und grundlegenden Freiheiten. ${ }^{34}$

\section{Der Verfassungsvorschlag vom 24.10.1997}

Der vierte Verfassungsvorschlag der Regierungskoalition PA vom $24.10 .1997^{35}$ bestätigte und vervollständigte den dritten vom 27.03.1997 in dessen wesentlichen Punkten, insbesondere im Hinblick auf das Konzept einer parlamentarischen Demokratie. Auch in den vierten Verfassungsvorschlag vom 24.10.1997 wurde ein Mechanismus zur Absicherung

Text unter: LAcNet (http://www.lacnet.org/srilanka/politics/devolution/proposal.html, AVL: 24.08.2000). Vgl. auch Seifert, F.-F., op.cit. (Fn. 7), S. 263-268 m.w.N.

Text unter: LAcNet (http://www.lacnet.org/srilanka/politics/devolution/proposal.html, AVL: 24.08.2000). Vgl. auch Seifert, F.-F., op.cit. (Fn. 7), S. 268-271 m.w.N. 24.08.2000). Vgl. auch Neelsen, J., op.cit. (Fn. 8), S. 23-29; Seifert, F.-F., op.cit. (Fn. 7), S. 271278 m.w.N. 
gegen Sezession aufgenommen ${ }^{36}$ und dabei die entsprechende Konstruktion des sich seinerseits auf den zweiten beziehenden dritten Verfassungsvorschlages bestätigt. Die Absicherung gegen Sezession war in Art. 1 (1), 1 (2), 2 (2) i.V.m. 220-223, 53, 54 Verfassungsvorschlag 24.10.1997 normiert.

Der Katalog von Menschenrechten und grundlegenden Freiheiten im dritten Kapitel des vierten Verfassungsvorschlages vom 24.10.1997 bestätigt grundsätzlich den des dritten vom 27.03.1997. ${ }^{37}$ Bedeutsam ist die Erweiterung des Kataloges um in vier Artikeln verfasste sogenannte "soziale" und "ökonomische" Rechte. ${ }^{38}$

Für die Umsetzung des beabsichtigten Autonomie-Konzeptes war das in Art. 127 Verfassungsvorschlag 24.10.1997 festgelegte Verfahren zum Aufbau des Systems der Regions bzw. Regional Councils von grösster Bedeutung. Das von zwei Referenden geprägte Verfahren hätte eine "re-demarcation" der verbundenen Nordöstlichen Provinz bewirkt und zu einer neuen Aufteilung dieser in verschiedene Regions geführt. Mit dem Verfahren und seinem möglichen Ergebnis glaubte die sri lankische Regierung, den Interessen der die aktuelle Siedlungsstruktur ${ }^{39}$ bildenden Bevölkerungsgruppen der früheren Östlichen Provinz weitgehend entsprechen zu können. Der Verfassungsvorschlag gründete sich

"on the fundamental principle that no decision should be imposed on the Eastern province without the full democratic consultation of the people of that province. The solution, taken together with the democratic process by which it is made, defines a set of choices which may not satisfy all in each of the three communities but can certainly satisfy the large majority." 40

Ursprünglich statuierte Art. 127 Verfassungsvorschlag 24.10.1997 ein vierstufiges Verfahren zum Aufbau des Systems der Regions und Regional Councils. ${ }^{41}$ Gemäss Art. 127 (1) i.V.m. Part B / First Schedule Verfassungsvorschlag 24.10.1997 sollten zunächst Regional Councils in den Regionen Central, North Central, North Western, Sabaragamuwa,

$$
\begin{aligned}
& \text { Vgl. Neelsen, J., op.cit. (Fn. 8), S. } 24 . \\
& \text { Vgl. Art. 8-25 Verfassungsvorschlag 24.10.1997 (S. 8-14 der offiziellen Publikation). }
\end{aligned}
$$

Gegenüber dem dritten Verfassungsvorschlag wurden in den vierten vom 24.10.1997 mehrere "Special rights of children" (Art. 22 Verfassungsvorschlag 24.10.1997), die "Freedom from forced labour" (Art. 23 Verfassungsvorschlag 24.10.1997), das "Right to safe conditions of work" (Art. 24 Verfassungsvorschlag 24.10.1997) und vier verschiedene "Social rights" (Art. 25 Verfassungsvorschlag 24.10.1997) aufgenommen.

Die Regierung ging von in der früheren Östlichen Provinz lebenden 41 v.H. Tamilen, 33 v.H. Muslimen und 26 v.H. Sinhalesen aus, vgl. The Sunday Observer, Political News Section, 19.10.1997 (http://www.lanka.net/lakehouse/1997/11/30/pol01.html, AVL: 22.10.1997).

Minister G. L. Peiris, zit. nach: The Sunday Observer, Political News Section, 19.10.1997 (http://www.lanka.net/lakehouse/1997/11/30/pol01.html, AVL: 22.10.1997).

Vgl. dazu die Darstellung bei Seifert, F.-F., op.cit. (Fn. 7), S. 276-278. 
Southern, Western und Uva errichtet werden, wobei das "Capital Territory" auszuklammern gewesen wäre, Art. 127 (5) i.V.m. Part A / First Schedule Verfassungsvorschlag 24.10.1997.

In einer zweiten Phase wären die Wahlberechtigten der "Administrative Districts" Trincomalee und Batticaloa aufgerufen gewesen, mittels eines Referendums über den Zusammenschluss der östlichen Verwaltungsdistrikte Trincomalee und Batticaloa mit den nördlichen Jaffna, Kilinochchi, Mannar, Mullaitivu und Vavuniya zu einer "North Eastern Region" zu entscheiden. Hätte sich die Mehrheit gegen einen Zusammenschluss entschieden, wären in einer dritten Phase gemäss Art. 127 (2) (b) (ii) i.V.m. Part D / First Schedule Verfassungsvorschlag 24.10.1997 zwei getrennte Regionen mit jeweils eigenen Regional Councils errichtet worden, zum einen die "Eastern Region", zum anderen die "Northern Region". Hätte sich die Mehrheit jedoch für einen Zusammenschluss entschieden, wäre es nach Art. 127 (2) (b) (i) i.V.m. Part C / First Schedule, 127 (3) i.V.m. Part C / First Schedule Verfassungsvorschlag 24.10.1997 zur Errichtung einer verbundenen "North Eastern Region" und zugleich zur Errichtung einer "South Eastern Region" gekommen. Dem hätte sich ein zweites Referendum angeschlossen, in dem die Wahlberechtigten des "Polling Division" Ampara zur Abstimmung darüber aufgerufen gewesen wären, ob eine eigenständige "Region of Ampara" errichtet werden solle, Art. 127 (4) (a) Verfassungsvorschlag 24.10.1997.

Hätte sich in der vierten und letzten Phase des Verfahrens zum Aufbau des Systems der Regionen und Regional Councils die Mehrheit gegen eine separate "Region of Ampara" entschieden, wäre der Wahlbezirk Ampara der Region Uva zugeordnet worden, Art. 127 (4) (b) (ii) i.V.m. Part B / First Schedule Verfassungsvorschlag 24.10.1997. Andernfalls hätte der Wahlbezirk Ampara eine eigenständige "Region of Ampara” gebildet, Art. 127 (4) (b) (i) i.V.m. Part E / First Schedule Verfassungsvorschlag 24.10.1997.

Als Ergebnis des Verfahrens waren also folgende drei Varianten einer Neuordnung denkbar: ${ }^{42}$ erstens die Aufhebung der Verbindung der Nordöstlichen Provinz und Errichtung einer "Eastern Region" "43 und einer "Northern Region", 44 deren jeweilige Territorien beizubehalten gewesen wären, zweitens die Errichtung einer "North Eastern Region", 45 einer

Vgl. dazu die Darstellungen bei Seifert, F.-F., op.cit. (Fn. 7), S. 332-336 (Darstellungen 6-9 in Anhang 2).

Bestehend aus den Verwaltungsdistrikten Trincomalee und Batticaloa, den Wahlbezirken Kalmunai, Samanthurai und Pottuvil und dem Wahlbezirk Ampara ("constituting the Administrative District of Ampara").

Bestehend aus den Verwaltungsdistrikten Jaffna, Kilinochchi, Mannar, Mullaitivu und Vavuniya.

45

Bestehend aus den (nördlichen) Verwaltungsdistrikten Jaffna, Kilinochchi, Mannar, Mullaitivu und Vavuniya sowie den (östlichen) Batticaloa und Trincomalee. 
"South Eastern Region",46 und einer eigenständigen "Region of Ampara"47 oder drittens die Errichtung einer "North Eastern Region", 48 einer "South Eastern Region"49 und einer "Uva Region", 50 der der Wahlbezirk Ampara zuzuordnen gewesen wäre. In allen drei Varianten sah die sri lankische Regierung die Bevölkerungsgruppen für ausreichend miteinander verknüpft, um einer Sezession insbesondere des nordöstlichen Staatsgebietes vorzubeugen. ${ }^{51}$

\section{Regelungsgegenstände des Verfassungsvorschlages vom 03.08.2000}

Mit dem Gesetzesentwurf vom 03.08.2000 - "An Act to Repeal and Replace the Constitution of the Socialist Republic of Sri Lanka (Bill No. 372)" - brachte die sri lankische Regierung einen vollständigen Verfassungstext zur Diskussion in das Parlament ein. ${ }^{52}$ Den 31 Kapiteln ist eine Präambel vorangestellt. Kapitel VII und VIII (Art. 55-76) Verfassungsvorschlag 03.08.2000 haben die zentrale Exekutive zum Regelungsgegenstand, Kapitel IX bis XI (Art. 77-102) Verfassungsvorschlag 03.08.2000 die zentrale Legislative, Kapitel XVII bis XIX (Art. 146-189) Verfassungsvorschlag 03.08.2000 die Judikative. In den Kapiteln XXVII bis XXXI (Art. 233-256) Verfassungsvorschlag 03.08.2000 werden die erforderlichen Übergangsbestimmungen getroffen, denen sich die Anhänge anschliessen. Die Staatsstruktur ist in Kapitel I (Art. 1-6) Verfassungsvorschlag 03.08.2000 geregelt, im darauf folgenden Kapitel II Verfassungsvorschlag 03.08.2000 wird dem Buddhismus "the foremost place" eingeräumt. Die Staatsstruktur wird auch von dem in Kapitel XV (Art. 127-141) Verfassungsvorschlag 03.08.2000 enthaltenen Autonomie-Konzept der "Devolution of Power to Regions" bestimmt, ebenso vom Demokratie-Konzept, welches in Kapitel XIII (Art. 106-121) Verfassungsvorschlag 03.08.2000 verankert ist. Kapitel III (Art. 8-31) Verfassungsvorschlag 03.08.2000 fasst die "Fundamental Rights and Freedoms" zusammen.

Bestehend aus den Wahlbezirken Kalmunai, Sammanthurai und Pottuvil. Ampara.

51 Vgl. The Sunday Observer, Political News Section, 19.10.1997 (http://www.lanka.net/lakehouse/ 1997/11/30/pol01.html, AVL: 22.10.1997).

52

Text unter: LacNet (http://www.lacnet.org/srilanka/politics/devolution/doc033.html, AVL: 24.08.2000).
} 


\section{Die Verfassungsreform und das Selbstbestimmungsrecht der Sri Lanka-Tamilen}

Die bisherigen vier Verfassungen Ceylons bzw. Sri Lankas blieben untauglich, das Selbstbestimmungsrecht der Sri Lanka-Tamilen derart angemessen zu berücksichtigen, dass sich das sri lanka-tamilische Volk in den sri lankischen Staatsverband integriert fühlen konnte, eher noch wurde es gerade durch das Machtmittel der Verfassung wieder und wieder ausgegrenzt. ${ }^{53}$ Nachfolgend werden die Regelungsgegenstände des aktuellen Verfassungsvorschlages vom 03.08.2000 betreffend das äussere (1) und innere (2) Selbstbestimmungsrecht des Volkes der Sri Lanka-Tamilen dargestellt und abschliessend einer Bewertung unterzogen (3).

\section{Regelungsgegenstände des Verfassungsvorschlages vom 03.08.2000 betreffend das äussere Selbstbestimmungsrecht}

Die sri lankische Regierung sieht offensichtlich die Notwendigkeit, in die Verfassung eine Absicherung gegen Sezessionsbestrebungen aufzunehmen (a), in diesem Zusammenhang ist auch auf die fehlende Anerkennung des sri lanka-tamilischen Volkes als eigenständiges Volk im ethnischen Sinn und damit möglichen Rechtsträger eines Selbstbestimmungsrechts zu verweisen (b).

\section{a. Verfassungsrechtlich verankerte Absicherung gegen Sezession}

Auch mit dem jüngsten Gesetzesvorschlag zur Verfassungsreform - "An Act to repeal and replace the Constitution of the Socialist Republic of Sri Lanka" - vom 03.08.2000 soll die Absicherung gegen eine mögliche Sezession wie schon in den früheren Vorschlägen verfassungsrechtlich verankert werden.

Bereits in Kapitel I (Art. 1-6) - "The People, The State and Sovereignty" - Verfassungsvorschlag 03.08.2000 ${ }^{54}$ wird unter Art. 1 (2) Verfassungsvorschlag 03.08.2000 die Pflicht des Staates - gemeint sind neben dem "Centre" auch die "Regional Administrations" und "Regional Councils", Art. 254 Verfassungsvorschlag 03.08.2000 - festgehalten, die territoriale Integrität der Republik Sri Lanka zu sichern, also eine Sezession zu verhindern:

53

54

Vgl. Neelsen, J., op.cit. (Fn. 8), S. 22 f, 29-31.

Text unter: LAcNet (http://www.lacnet.org/srilanka/politics/devolution/doc033b.html, AVL: 24.08.2000). 
"The State shall safeguard the independence, sovereignty, unity and the territorial integrity of the Republic and shall preserve and advance a Sri Lankan identity, recognizing the multi-ethnic, multi-lingual and multi-religious character of Sri Lankan society."

Dem entspricht das im selben Kapitel unter Art. 3 (2) Verfassungsvorschlag 03.08.2000 statuierte Verbot eines Regional Council oder einer Regional Administration, auf eine Sezession hinzuwirken:

"Any Regional Council or Regional Administration shall not, by direct or indirect means, promote or otherwise advocate or attempt to promote or otherwise advocate an initiative towards -

(a) the separation or secession of any Region or part thereof, from the Republic;

(b) the alteration of the area or boundaries of a Region;

(c) the alteration of the name of a Region;

(d) the formation of a new Region by separation of territory from any Region or by uniting two or more Regions or parts of Regions or by uniting any territory with a part of any Region."

Mit der Absicherung gegen eine Sezession in Kapitel I Verfassungsvorschlag 03.08.2000 stehen vor allem die Bestimmungen in Kapitel XXIII (Art. 219-224) Verfassungsvorschlag 03.08.2000 ${ }^{55}$ zur "Public Security" im Zusammenhang:

"Assumption of powers by the President.

223. (1) Where the President, upon being advised by the Prime Minister, is of opinion that a situation has arisen in which a Regional Administration is promoting armed rebellion or insurrection or engaging in an intentional violation of Article 1 or 2 or 3, or the provisions of Chapter XV or Chapter XXII of the Constitution which constitutes a clear and present danger to the unity and sovereignty of the Republic, the President may, by Proclamation -

(a) assume to the President, all or any of the functions of the administration of the Region and all or any of the powers vested in, or exercisable by, the Governor, the Chief Minister, the Board of Ministers or any body or authority in the Region; and

(b) where it is necessary for the effectual exercise of the powers under sub-paragraph (a) of this paragraph, dissolve the Regional Council."

Damit werden dem Präsidenten zur Aufrechterhaltung der "Public Security" weitreichende Befugnisse an die Hand gegeben, sollte sich eine Sezession anbahnen. "Unity and sovereignty" werden von der den Vorschlag unterbreitenden sri lankischen Regierung als "sacrosanct principles" angesehen; insbesondere die Befugnisse des Präsidenten zur Auf- 
rechterhaltung der "Public Security" sollen die Befürchtung, eine Region könne sich selbst zu einer "separate nation" erklären, als "totally erroneous conception" erscheinen lassen insoweit werden die Vorschläge vom 16.01.1996, vom 27.03.1997 und vom 24.10.1997 bestätigt. $^{56}$

Kapitel VI (Art. 52-54) Verfassungsvorschlag 03.08.2000 ${ }^{57}$ enthält eine Zusammenstellung der "Principles of State Policy and Fundamental Duties". In Art. 52 (1) Verfassungsvorschlag 03.08.2000 wird dem Staat aufgegeben, die "national unity" zu fördern:

"The State shall recognize the pluralistic character of Sri Lankan society and strengthen national unity by ensuring that the composition of the institutions of State reflects such character and shall promote cooperation and mutual trust, confidence and understanding among all sections of the People of Sri Lanka."

Dagegen werden dem Bürger Sri Lankas in Art. 53 (1), (3) Verfassungsvorschlag 03.08.2000 "fundamental duties" auferlegt, insbesondere, die territoriale Integrität Sri Lankas zu wahren und zu schützen:

"It shall be the duty of every citizen to -

(1) uphold and protect the independence, sovereignty, unity and territorial integrity of Sri Lanka; ...

(3) foster national unity and promote harmony amongst all the People of Sri Lanka”.

Auch wenn in der beabsichtigten Verfassung die territoriale Integrität des Inselstaates noch so oft beschworen wird - eine etablierte Sezessionsbewegung wird sich kaum von verfassungsrechtlich verankerten Sezessionsverboten beeindrucken lassen, sich über solche vielmehr schlicht hinwegsetzen.

\section{b. $\quad$ Keine Anerkennung der Sri Lanka-Tamilen als eigenständiges Volk im ethnischen Sinn}

Im gesamten Verfassungsvorschlag vom 03.08.2000 wird davon abgesehen, auf die tatsächliche Bevölkerungsstruktur Sri Lankas einzugehen und dabei die Sri Lanka-Tamilen als eigenständiges Volk im ethnischen Sinn - gerade neben dem der Sinhalesen - zu nennen. Grundsätzlich ist im Verfassungsvorschlag das "people" als Staatsvolk genannt, gemäss Art. 1 (2) Verfassungsvorschlag 03.08.2000 hat der Staat"the multi-ethnic, multi-lingual and multi-religious character of Sri Lankan society", nach Art. 52 (1) Verfassungsvorschlag 03.08.2000 "the pluralistic character of Sri Lankan society" anzuerkennen; in letz-

56

57

Vgl. Seifert, F.-F., op.cit. (Fn. 7), S. 265 f, 269, 272 m.w.N.

Text unter: LAcNet (htp.//www.lacnet.org/srilanka/politics/devolution/doc033g.html, AVL: 24.08.2000). 
terer Bestimmung findet sich auch die Formulierung "sections of the People of Sri Lanka". Im Rahmen der Bestimmungen über den "Interim Council for the Northern and Eastern Regions" werden in Art. 245, 246 Verfassungsvorschlag 03.08.2000 die "communities" beider Regionen genannt und dabei auf die "second and third major communities" verwiesen. In Art. 251 und 252 Verfassungsvorschlag 03.08.2000 werden dementsprechend die "three major communities in the Northern and Eastern Regions" anerkannt.

Auf den Charakter der Gesamtheit der Sri Lanka-Tamilen als Volk im ethnischen Sinn mit einer nationalen Identität zu verweisen, wird im Verfassungsvorschlag sorgfältig vermieden. Auch das Autonomie-Konzept der "Union of Regions" stellt nicht auf das Siedlungsgebiet der Sri Lanka-Tamilen ab, so dass es einer Anerkennung der Sri Lanka-Tamilen als eigenständiges Volk im ethnischen Sinn scheinbar auch nicht bedarf. Damit wird das Bemühen der sri lankischen Regierung deutlich, dem Vorwurf einer Begünstigung sezessionistischer Bestrebungen eines Bevölkerungsteiles von vornherein entgegenzutreten.

\section{Regelungsgegenstände des Verfassungsvorschlages vom 03.08.2000 betreffend das innere Selbstbestimmungsrecht}

Entsprechend den bereits genannten Ebenen innerer Selbstbestimmung wird nachfolgend der ersten Ebene zuzuordnen - der Katalog von Menschenrechten und grundlegenden Freiheiten vorgestellt (a), sodann das Autonomie-Konzept der beabsichtigten Union of Regions (b) und letztlich das Demokratie-Konzept (c).

a.

Der Katalog von Menschenrechten und grundlegenden Freiheiten

Kapitel III Verfassungsvorschlag 03.08.2000 enthält einen Katalog von Fundamental Rights and Freedoms. ${ }^{58}$ Das Diskriminierungsverbot nach Art. 11 (2) (a) Verfassungsvorschlag 03.08.2000 beispielsweise verbietet eine Ungleichbehandlung "on the grounds of ethnicity, religion, language, caste, gender, sex, political or other opinion, national or social origin, place of birth, mode of acquisition of citizenship, marital status, maternity, parental status or any one of such grounds". Art. 19 (1) Verfassungsvorschlag 03.08.2000 bestimmt: "Every citizen is entitled alone or in association with others to enjoy and promote such citizen's own culture and to use such citizen's own language." Zu beachten sind allerdings die weit gefassten Vorbehalte bestimmter grundrechtlicher Verbürgungen ${ }^{59}$ und die Mög-

Text unter: LAcNet (http://www.lacnet.org/srilanka/politics/devolution/doc033d.html, AVL: 24.08.2000). Vgl. auch Art. 2 (2) (d) Verfassungsvorschlag 03.08.2000.

59

Vgl. Art. 10 (16) (b), 11 (5), 12 (3), 14 (2), 15 (4), 16 (2), 17 (2), 18 (3), 19 (2), 20 (2) Verfassungsvorschlag 03.08.2000. 
lichkeit der "Derogation in times of public emergency" gemäss Art. 27 Verfassungsvorschlag 03.08.2000.

\section{b. Das Autonomie-Konzept}

Mit dem Vorschlag für eine neue Verfassung vom 03.08.2000 gab die sri lankische Regierung das vierstufige Verfahren des Verfassungsvorschlages vom 24.10.1997 auf. Damit ist auch die Möglichkeit der Errichtung einer eigenständigen "Region of Ampara" entfallen.

Der aktuelle Gesetzesvorschlag vom 03.08.2000 sieht in Kapitel XV (Art. 127-141) - "The devolution of power to regions" - Verfassungsvorschlag 03.08.2000 60 nur noch ein Referendum der Wahlberechtigten der östlichen Verwaltungsdistrikte Ampara, Batticaloa und Trincomalee über die Frage eines Zusammenschlusses dieser mit den nördlichen Verwaltungsdistrikten Jaffna, Kilinochchi, Mannar, Mullaitivu und Vavuniya zu einer "NorthEastern Region" vor: ${ }^{61}$

\section{"Establishment of Regional Council}

127. (1) (a) There shall be a Regional Council for every Region specified in Part A of the First Schedule.

... (2) (a) The President shall, by Order published in the Gazette, require a Referendum to be held in the Administrative Districts of Trincomalee, Batticaloa and Amparai, and fix a date or dates therefore being a date or dates not earlier than the expiration of a period of nine years from the commencement of the Constitution and not later than three month prior to the expiration of a period of ten years from the commencement of the constitution to enable the electors of such Districts to decide on the question whether or not such Districts and the Administrative Districts of Jaffna, Kilinochchi, Vavuniya, Mannar und Mullaitivu should form one Region to be designated the NorthEastern Region."

Weiter bestimmt Art. 127 (2) (b) Verfassungsvorschlag 03.08.2000:

60

Text unter: LAcNet (http://www.lacnet.org/srilanka/politics/devolution/doc033p.html, AVL: 24.08.2000).

61 Der "First Schedule" Verfassungsvorschlag 03.08.2000 bestimmt in "Part A" als Regionen "Western" (Verwaltungsbezirke Colombo, Gampaha, Kalutara), "Central" (Kandy, Nuwara Eliya, Matale), "Southern" (Galle, Matara, Hambantota), "North Central" (Anuradhapura, Polonnaruwa), "North Western" (Puttalam, Kurunegala), "Sabaragamuwa" (Ratnapura, Kegalle), "Uva" (Badulla, Moneragala), in "Part B" als Region "North-Eastern" (Verwaltungsbezirke Jaffna, Kilinochchi, Mannar, Vavuniya, Mullaitivu und Verwaltungsbezirke Trincomalee, Batticaloa, Ampara) und in "Part C" als Regionen "Northern" (Verwaltungsbezirke Jaffna, Kilinochchi, Mannar, Vavuniya, Mullaitivu) und "Eastern" (Verwaltungsbezirke Trincomalee, Batticaloa, Ampara). 
"Where at such Referendum the question

(i) is answered in the affirmative by a majority of the valid votes cast, a Regional Council shall be established for the North-Eastern Region specified in Part B of the First Schedule with effect from such date as the President shall appoint by Order published in the Gazette;

(ii) is not answered in the affirmative, two separate Regional Councils shall be established for the Northern Region and the Eastern Region as specified respectively in Part C of the First Schedule with effect from such date as the President shall appoint by Order published in the Gazette."

Als Ergebnis des Referendums in den drei östlichen Distrikten ist daher nur noch entweder die Errichtung einer verbundenen "North-Eastern Region"62 oder die Errichtung zweier eigenständiger Regionen, der "Northern Region"63 zum einen, der "Eastern Region"64 zum anderen, denkbar.

Das nunmehr beabsichtigte Referendum über einen Zusammenschluss von Nördlicher und Östlicher Region wird aus den Reihen der Vertreter sri lanka-tamilischer Interessen abgelehnt. Als Alternative zu einem unabhängigen sri lanka-tamilisch dominierten Staat "Tamil Eelam" müsse zumindest eine verbundene "North-Eastern Region" die "traditional Tamil homelands" im Nordosten des Inselstaates widerspiegeln. ${ }^{65}$ Bei einem Referendum der östlichen Verwaltungsdistrikte Ampara, Batticaloa und Trincomalee stünde jedoch zu befürchten, dass wegen der Mehrheit von Sinhalesen und Muslimen ein Zusammenschluss abgelehnt werden würde. Demgegenüber hätte mit dem vierstufigen Verfahren des vorhergehenden Verfassungsvorschlages zumindest die Möglichkeit bestanden, gerade den Distrikt Ampara mit seiner Sinhalesen- und Muslim-Mehrheit auszugliedern und Nördliche und Östliche Region gleichwohl zusammenzuschliessen. Die Abkehr vom Autonomie-Konzept im Verfassungsvorschlag vom 24.10.1997 kann daher gleichfalls als Rückschritt bewertet werden, das innere Selbstbestimmungsrecht der Sri Lanka-Tamilen zu verwirklichen und damit letztlich den ethnischen Konflikt beizulegen.

Über die Möglichkeit zweier dauerhaft getrennter Regionen, der Nördlichen einerseits und der Östlichen andererseits, kann auch nicht die übergangsweise Bildung eines ’Interim

Bestehend aus den Verwaltungsbezirken Jaffna, Kilinochchi, Mannar, Vavuniya, Mullaitivu und den Verwaltungsbezirken Trincomalee, Batticaloa, Ampara.

Bestehend aus den Verwaltungsbezirken Jaffna, Kilinochchi, Mannar, Vavuniya und Mullaitivu.

64

65 Bestehend aus den Verwaltungsbezirken Trincomalee, Batticaloa und Ampara.

Vgl. Keller, W., Eine neue Verfassung muss auch für Minderheiten akzeptabel sein, in: Südasien 20 (2000), Nr. 5, S. 11. 
Council for the Northern and Eastern Regions" in Kapitel XXVIII (Art. 243-253) Verfassungsvorschlag 03.08.2000 ${ }^{66}$ für einen Zeitraum von fünf Jahren hinwegtäuschen. ${ }^{67}$

Dem nach Art. 127 Verfassungsvorschlag 03.08.2000 errichteten Regional Council kommt Gesetzgebungszuständigkeit nach Art. 137 i.V.m. List II - Regional List Verfassungsvorschlag 03.08.2000 zu: ${ }^{68}$

"Legislative power, delegation and procedure.

137. (1) Subject to the provisions of the Constitution, the Regional Council of a Region shall have exclusive power to make Statutes, including Statutes having retrospective effect, for such Region or any part thereof with respect to any of the matters enumerated in the 'Regional List'."

Text unter: LacNet (http://www.lacnet.org/srilanka/politics/devolution/doc033zc.html, AVL: 24.08.2000).

Art. 243 (1) Verfassungsvorschlag 03.08.2000 bestimmt: "There shall be an Interim Council for the Northern and Eastern Regions set out in Part C of the First Schedule, with effect from the commencement of the Constitution, and shall continue for a period of five years from that date and such Interim Council shall be deemed to have been dissolved at the end of that period."

Als "Powers of Interim Council and Board of Ministers" statuiert Art. 245 Verfassungsvorschlag 03.08.2000: "Subject to the provisions of this Chapter, the Governor of the Northern and Eastern Regions, the Interim Council and the Board of Ministers appointed under this Chapter shall exercise and perform, the powers and duties conferred and imposed respectively on, and discharge the functions assigned respectively to, the Governor of a Region, a Regional Council and the Board of Ministers of a Region, by this Constitution, and all the provisions of this Constitution relating to the Governor of a Region, Regional Councils and the Board of Ministers of a Region shall, save as expressly provided in this Chapter, apply respectively, to the Governor of the Northern and Eastern Regions and to the Interim Council and Board of Ministers appointed under this Chapter. (2) The Governor, Interim Council and the Board of Ministers shall exercise their respective powers with rigorous impartiality on behalf of all the people in the two Regions, recognizing the diversity of their identities and traditions, and such exercise shall be founded on the principles of full respect for the equality of the civil, political, social, religious and cultural rights of those people and of the freedom from discrimination for all citizens, and on parity of esteem and equal treatment for the identity, ethos and aspirations of all communities in the two Regions."

Dem "Interim Council" werden in Art. 246, 250 Verfassungsvorschlag 03.08.2000 bestimmte legislative Kompetenzen eingeräumt. Neben dem "Interim Council" sollen für die beiden Provinzen ein "Board of Ministers", ein "Interim Regional Council", eine "Public Service Commission", eine "Police Commission", eine "Finance Commission", "Cultural Committees" und eine "Equality Commission" aufgestellt werden, Art. 244, 253, 247, 248, 249, 251, 252 Verfassungsvorschlag 03.08.2000.

Text der "List II - Regional List" Zweiter Anhang Verfassungsvorschlag 03.08.2000 unter: LacNet (http://www.lacnet.org/srilanka/politics/devolution/doc033zg.html, AVL: 24.08.2000); die "Regional List" enthält 65 Regelungsgegenstände (Beispiele: "9. Motor vehicle licence fees."; "34. Markets and fairs."; "58. Sports."). Zum "Regional Council" vgl. auch Art. 128, 132, 133 Verfassungsvorschlag 03.08.2000. 
Davon ist die Gesetzgebungszuständigkeit des zentralen Parlaments nach Art. 91 i.V.m. List I - Reserved List, 92 Verfassungsvorschlag 03.08.2000 abzugrenzen: ${ }^{69}$

"Legislative power.

91. (1) Parliament has exclusive power to make laws, for the whole or any part of the territory of the Republic, including laws having retrospective effect, with respect to any of the matters enumerated in List I of the Second Schedule (referred to as the 'Reserved List')....

Delegation of legislative power.

92. (1) Parliament shall not abdicate or in any manner alienate its legislative power and shall not set up any authority with any such legislative power."

Die "executive powers of the Region" übt ein "Governor" aus, Art. 130, 129 Verfassungsvorschlag 03.08.2000, der dabei von einem "Board of Ministers", welchem je Ministerium bestimmte "Executive Committees" zugeordnet sind und dem ein "Chief Minister" vorsteht, unterstützt wird, Art. 134, 135 Verfassungsvorschlag 03.08.2000. Vornehmlich beratende Funktion kommt auch dem "Advocate-General of the Region" zu, Art. 136 Verfassungsvorschlag 03.08.2000. Gemäss Art. 141 Verfassungsvorschlag 03.08.2000 soll eine "Chief Ministers' Conference", die sich aus den "Chief Ministers" aller Regionen zusammensetzt, unter anderem dafür Sorge tragen, die regionalen Politiken zu koordinieren, in jeder Hinsicht Einvernehmen zwischen den Regionen herzustellen und deren Zusammenarbeit zu fördern.

\section{c. Das Demokratie-Konzept}

Nach Art. 52 (3) Verfassungsvorschlag $03.08 .2000^{71}$ soll der Staat "safeguard and strengthen the democratic structure of government and the democratic rights of the People".

Text der "List I - Reserved List" Zweiter Anhang Verfassungsvorschlag 03.08.2000 unter: LacNet (http://www.lacnet.org/srilanka/politics/devolution/doc033zf.html, AVL: 24.08.2000); die "Reserved List" enthält 86 Regelungsgegenstände (Beispiele: "1. Defence; national security; national police; security forces; special forces; para-military forces established by or under law."; "5. Foreign affairs, including all matters which bring the Republic of Sri Lanka into relations with other States and the undertaking of international obligations."; "86. Any other matter not enumerated in the Regional List.").

70 Text unter: LAcNet (http://www.lacnet.org/srilanka/politics/devolution/doc033k.html, AVL: 24.08.2000). 
Art. 2 - "Sovereignty of the People" - Verfassungsvorschlag 03.08.2000 ${ }^{72}$ bestimmt:

"(1) In the Republic, Sovereignty is in the People and is inalienable.

(2) Sovereignty includes powers of government, fundamental rights and the franchise and shall be exercised in the following manner:

(a) the legislative power of the Power of the People shall be exercised, by the People at a Referendum, by Parliament and by Regional Councils to the respective extends and in the manner hereinafter provided".

Nach Kapitel XII (Art. 103-105) - "The Referendum" - Verfassungsvorschlag 03.08.2000 sind den Wahlberechtigten bestimmte Gegenstände zur Entscheidung mittels Referendums vorzulegen, ${ }^{73}$ nach Art. 104 Verfassungsvorschlag 03.08.2000 insbesondere solche von "national importance" nach Ansicht des Präsidenten. Das sich aus 225 Abgeordneten zusammensetzende Parlament soll nach einem Wahlsystem nach deutschem Vorbild gewählt werden, vgl. Art. 116-118 Verfassungsvorschlag 03.08.2000. ${ }^{74}$ Die "legislative power" des zentralen Parlaments ist in Art. 91, 92 Verfassungsvorschlag 03.08.2000 statuiert. Das Parlament wählt den Präsidenten der Republik als Staatsoberhaupt, Art. 56, 55, 58 Verfassungsvorschlag 03.08.2000, ${ }^{75}$ der wiederum die Minister und den Premierminister ernennt, Art. 58 (d), 65 (2) (b) Verfassungsvorschlag 03.08.2000. ${ }^{76}$

\section{Bewertung}

Der Verfassungsvorschlag vom 03.08.2000 berücksichtigt das Selbstbestimmungsrecht des Volkes der Sri Lanka-Tamilen nicht in angemessener Weise und wird die Beilegung des ethnischen Konfliktes daher nicht fördern. So dezidiert mit dem Gesetzesentwurf die territoriale Integrität Sri Lankas gesichert und jegliche Sezessionsbestrebungen verboten werden, so untauglich wird das Autonomie-Konzept, das Volk der Sri Lanka-Tamilen in den Staatsverband zu integrieren, bleiben.

72

74

76 24.08.2000)

Text unter: LAcNet (http://www.lacnet.org/srilanka/politics/devolution/doc033m.html, AVL: 24.08.2000).

Kapitel XIII (Art. 106-121) - "The Franchise and Elections" - Verfassungsvorschlag 03.08.2000, Text unter: LAcNet (http://www.lacnet.org/srilanka/politics/devolution/doc033n.html, AVL: 24.08.2000).

Kapitel VII (Art. 55-64) - "The Central Executive - The President of the Republic" - Verfassungsvorschlag 03.08.2000, Text unter: LAcNet (http://www.lacnet.org/srilanka/politics/devolution/doc033h.html, AVL: 24.08.2000).

Kapitel VIII (Art. 65-76) - "The Central Executive - The President and the Cabinet of Ministers" - Verfassungsvorschlag 03.08.2000, Text unter: LAcNet (http://www.lacnet.org/srilanka/politics/ devolution/doc033i.html, AVL: 24.08.2000). 
Die beabsichtigte Union of Regions führt nicht zur Errichtung einer föderalen Staatsstruktur; eine dahingehende Terminologie im Verfassungsvorschlag wurde ganz bewusst von vornherein ausgeklammert. ${ }^{77}$ Aber auch in materieller Hinsicht bleibt der zentralistische Einheitsstaat Hauptmerkmal des Verfassungsvorschlages. Schon die politisch-territoriale Neuaufgliederung des Staatsgebietes in Regionen dient offensichtlich allein der Kontrolle der Forderung des sri lanka-tamilischen Volkes nach Selbstbestimmung. Zu der möglichen dauerhaften Zusammenlegung von Nördlicher und Östlicher Region wird es kaum kommen, zu Recht sieht J.P. Neelsen schon im vorhergehenden Verfassungsentwurf in der Neuaufgliederung eine "Beschneidung des [sri lanka-tamilischen] Herrschaftsbereiches durch ethnisch homogene Parzellierung". 78 Die mit dem Autonomie-Konzept beabsichtigte blosse symmetrische Dezentralisierung entbehrt jeglicher substantieller Kompetenzzuweisung von Regelungsgegenständen zur Selbstverwaltung an die Regionen. Die materiellen Befugnisse sind auf die unmittelbarsten regionalen bzw. lokalen Belange begrenzt. "[W]eit davon entfernt, selbstbestimmte und eigenverantwortliche sozial-territoriale Gebietskörperschaften zu werden, sind die Regionen zu reinen Lokaladministrationen bzw. Exekutivorganen der Zentralregierung geschrumpft." ${ }^{79}$ Dem entspricht zudem eine Verschiebung der Kompetenzverteilung zugunsten der Exekutive innerhalb einer Region; der jeweilige Regional Council kann den vom Präsidenten abhängigen und mit erheblicher Machtfülle ausgestatteten Gouverneur einer Region nicht kontrollieren, wobei auch das Board of Ministers kein Gegengewicht bildet.

Derart minimale Zugeständnisse der sinhalesisch dominierten Regierung in Form von bloss geringfügiger Dezentralisierung werden nicht ausreichen, der aus den Reihen des sri lankatamilischen Volkes erhobenen Sezessionsforderung die Grundlage zu entziehen. Ein angemessener Kompromiss wurde auch mit dem Verfassungsvorschlag vom 03.08.2000 noch nicht gefunden.

\section{Ausblick}

Die zuletzt regierende Koalition, die "People's Alliance" (PA) unter Führung von Präsidentin Chandrika Bandaranaike-Kumaratunga, konnte ihre Absicht, den Entwurf einer neuen Verfassung noch vor Ablauf der Legislaturperiode im August des Jahres 2000 im Parlament beraten und mit der erforderlichen parlamentarischen Zwei-Drittel-Mehrheit annehmen zu lassen, nicht mehr verwirklichen. Die Regierungskoalition sah sich gezwungen, den Verfassungsvorschlag vom 03.08.2000 wegen des inner- und ausserparlamentari-

Vgl. The Sunday Times, The Political Column, 31.12.2000 (http://www.is.lk/times/001231/ pol.html, AVL: 02.01.01).

78

79

Neelsen, J., op.cit. (Fn. 8), S. 26.

Neelsen, J., op.cit. (Fn. 8), S. 28. 
schen Widerstandes gegen das Reformvorhaben nur einen Tag, nachdem die Vorlage in das Parlament eingebracht wurde, zurückzuziehen. ${ }^{80}$

Wegen des Scheiterns des Reformvorhabens wurden das Parlament vorzeitig aufgelöst und Neuwahlen angesetzt. ${ }^{81}$ Bereits im Vorfeld dieser erklärte die Präsidentin, im Fall der Wiederwahl der Regierungskoalition würde der Verfassungsvorschlag erneut zur Debatte eingebracht, das Parlament möglicherweise sogar zu einer verfassungsgebenden Versammlung berufen. ${ }^{82}$ Bei den Parlamentswahlen konnte jedoch zunächst weder das Wahlbündnis der PA noch die oppositionelle "United National Party" (UNP) die absolute Mehrheit der Stimmen erringen. ${ }^{83}$ Da sie sich die Unterstützung anderer Parteien sichern konnte, stellt die PA nunmehr wiederholt die Regierung Sri Lankas unter Präsidentin Bandaranaike-Kumaratunga. Zur Bildung einer grossen Koalition mit der UNP als stärkster Oppositionspartei kam es dagegen nicht.

Es bleibt daher vorerst abzuwarten, wie die für eine Verfassungsreform erforderliche parlamentarische Zwei-Drittel-Mehrheit gesichert werden soll. Einerseits zielt das Agieren der PA und der UNP unter dem Banner eines "Common Programme for New Political Culture" auf einen breiten parlamentarischen Konsens ab, der möglicherweise im Rahmen einer Allparteienkonferenz gefunden werden soll. ${ }^{84}$ Andererseits erscheint die Vorstellung der Regierungskoalition, mittels Einberufung einer verfassungsgebenden Versammlung, die mit einfacher Mehrheit entscheiden würde, sodann der Annahme durch das Wahlvolk mittels

Vgl. Keller, W., Das Scheitern einer Präsidentin - Sri Lanka kann sich weiterhin nicht auf einen neuen Verfassungsentwurf einigen, in: Südasien 20 (2000), Nr. 5, S. 8-11; The Sunday Times, The Political Column, 13.08.2000 (http://www.lacnet.org/suntimes/000813/pol.html, AVL: 27.11.2000).

Vgl. The Sunday Times, The Political Column, 20.08.2000 (http://www.lacnet.org/suntimes/ 000820/pol.html, AVL: 27.11.2000).

Vgl. The Sunday Times, The Political Column, 08.10.2000 (http://www.lacnet.org/suntimes/ 001008/pol.html, AVL: 14.11.2000); ebd., News / Comment, 08.10.2000 (http://www.lacnet.org/ suntimes/001008/news2.html, AVL: 14.11.2000); ebd., The Political Column, 05.11.2000 (http:// www.lacnet.org/suntimes/001105/pol.html, AVL: 14.11.2000).

$\mathrm{Zu}$ den Wahlen und zum Wahlergebnis vgl. Keller, W., Eine zweite Chance - Die ,Peoples Alliance' gewinnt erneut die Parlamentswahlen, in: Südasien 20 (2000), Nrn. 6-7, S. 7-9; The Sunday Times, The Political Column, 15.10.2000 (http://www.lacnet.org/suntimes/001015/pol. html, AVL: 14.11.2000); ebd., News / Comment, 15.10.2000 (http://www.lacnet.org/suntimes/ 001015/newsm.html, AVL: 14.11.2000); ebd., The Political Column, 22.10.2000 (http://www. lacnet.org/suntimes/001022/pol.html, AVL: 14.11.2000).

Vgl. The Sunday Times, The Political Column, 05.11.2000 (http://www.lacnet.org/suntimes/ 001105/pol.html, AVL: 14.11.2000), 12.11.2000 (http://www.lacnet.org/suntimes/001112/pol. html, AVL: 14.11.2000), 19.11.2000 (http://www.lacnet.org/suntimes/001119/pol.html, AVL: 22.11.2000), 29.10.2000 (http://www.lacnet.org/suntimes/001029/pol.html, AVL: 14.11.2000). 
Referendums und sich anschliessender präsidentieller Proklamation, ${ }^{85}$ das Erfordernis einer parlamentarischen Zwei-Drittel-Mehrheit umgehen zu können, als verfassungsrechtlich bedenklich, zumal sich die oppositionelle UNP kaum auf ein derartiges Vorgehen einlassen wird. $^{86}$

Die bereits 1995 angestossene Verfassungsreform in Sri Lanka droht damit wiederholt eher zum Gegenstand parteienpolitischer Interessen innerhalb der einen, sinhalesischen, Konfliktpartei zu werden, als letztlich zumindest auch der Beilegung des sinhalesisch - sri lanka-tamilischen Konfliktes zu dienen. ${ }^{87}$ Ist aber schon die Verwirklichung der Verfassungsreform mehr als fraglich - Minister G.L. Peiris äusserte bereits, "that promulgating a new constitution was not the priority of the government now" ${ }^{, 8}-$, bleibt immer noch $\mathrm{zu}$ bedenken, dass sich das neue Regelwerk auch rechtspraktisch bewähren muss. Ohne die Akzeptanz der LTTE-Guerilla als massgeblicher Vertreter des Volkes der Sri Lanka-Tamilen als der anderen Konfliktpartei ist dies ausgeschlossen. Ob die eingangs erwähnten zwischen der sri lankischen Regierung und der LTTE beabsichtigten Gespräche tatsächlich zu einer Annahme der Verfassungsreform auch auf sri lanka-tamilischer Seite führen, ist völlig offen und - bei gehöriger Berücksichtigung der Ergebnisse bisheriger Reformvorhaben - eher zweifelhaft.

Ein Scheitern schon der Verfassungsreform selbst oder deren späterer Umsetzung jedoch würde dem sri lanka-tamilischen Volk innere Selbstbestimmung weiterhin vorenthalten, einen Fortgang des opferreichen ethnischen Konfliktes zwischen Sinhalesen und Sri LankaTamilen bedeuten und zumindest völkerrechtstheoretisch Argumente für eine Sezession zur Errichtung eines "Tamil Eelam" liefern.

Vgl. dazu den Zusatz am Ende Verfassungsvorschlag 03.08.2000, Text unter: LAcNet (http:// www.lacnet.org/srilanka/politics/devolution/doc033zi.html, AVL: 02.01.2001).

Vgl. The Sunday Times, The Political Column, 05.11.2000 (http://www.lacnet.org/suntimes/ 001105/pol.html, AVL: 14.11.2000); ebd., 24.12.2000 (http://www.is.lk/times/001224/pol.html, AVL: 02.01.2001).

Vgl. The Sunday Times, The Political Column, 31.12.2000 (http://www.is.lk/times/001231/pol. html, AVL: 02.01.2001).

"He said the new constitution would be promulgated only after obtaining the UNP support as the process required a two-thirds majority in Parliament.", zit. nach: The Sunday Times, The Political Column, 10.12.2000 (http://www.lacnet.org/suntimes/001210/pol.html, AVL: 11.12.2000); vgl. auch ebd., 24.12.2000 (http://www.is.lk/times/001224/pol.html, AVL: 02.01.01), abzuwarten ist daher, ob die neue Verfassung tatsächlich, wie von Präsidentin Chandrika Bandaranaike-Kumaratunga in Aussicht gestellt, im Jahr 2001 durchgesetzt wird. 